\section{Minimas Alterações Hormonais em Paciente Com Grande Feocromocitoma}

\section{RESUMO}

Relatamos caso clínico no qual o paciente apresentou sintomas sugestivos de feocromocitoma, grande tumor (maior que $50 \mathrm{~g}$ ) e mínima alteração laboratorial, exemplificando uma armadilha diagnóstica. Um homem de 31 anos apresentou dois episódios de abdômen agudo, sendo o último acompanhado por cefaléia, hipertensão arterial, rubor facial, sudorese e palidez cutânea. Em outra internação, o paciente apresentava hipertensão arterial sustentada e arritmia cardíaca. Em relação aos testes laboratoriais, apenas o ácido vanil-mandélico foi levemente alterado. Cintilografia com MIBG foi realizada e sugeriu a presença de grande massa adrenal compativel com feocromocitoma. Uma amostra histopatológica da peça foi obtida após cirurgia e confirmou esta hipótese. Esse caso sugere que em pacientes que possuem sintomas sugestivos de feocromocitoma, mesmo com valores normais de catecolaminas plasmáticas e metanefrinas urinárias, devemos considerar as possibilidades de um grande tumor metabolizando catecolaminas em seu interior ou que sofreu necrose hemorrágica. (Arq Bras Endocrinol Metab 2006;50/1:145-149)

Descritores: Feocromocitoma; Hipertensão; Catecolaminas; Metanefrinas; Neoplasia endócrina múltipla

\begin{abstract}
Slight Hormonal Alterations in a Patient With a Large Pheochromocytoma. We present a case report that the patient had symptoms suggesting pheochromocytoma, a large tumor (>50 g) and a single minimally altered laboratorial test, exemplifying a diagnostic pitfall. A 31y.o. male patient had two acute abdominal events, the last one accompanied by headache, arterial hypertension, facial flushing, perspiration and cutaneous pallor. In another admission, the patient had sustained arterial hypertension and cardiac arrhythmia. From laboratory analysis, the vanililmandelic acid was slightly modified. Scintigraphy disclosed a large adrenal mass suggesting pheochromocytoma. Histopathology confirmed this hypothesis. This report points out that patients with symptoms suggesting pheochromocytoma, even when plasma catecholamines and urinary metanephrines levels are normal, may harbor large tumors with a high catecholamines turnover or that had undergone hemorrhagic necrosis. (Arq Bras Endocrinol Metab 2006; 50/1:145-149)
\end{abstract}

Keywords: Pheochromocytoma; Hypertension; Catecholamines; Metanephrines; Multiple endocrine neoplasia

$\mathbf{F}^{\mathrm{s}}$ OCROMOCITOMA é uma causa importante de hipertensão sustentada ou intermitente, resistente ao tratamento clínico convencional (1), sendo responsável por aproximadamente $0,2 \%$ dos casos de hipertensão arterial sistêmica $(2,3)$. apresentação de caso

\author{
Josivan G. de Lima \\ Francisco Bandeiva \\ Cristina H. Bandeira \\ Maurício de Paula \\ Maria L.C. Nóbrega \\ Saul O. e Costa \\ Lúcia H.C. Nóbrega
}

Disciplina de Endocrinologia do Departamento de Medicina

Clinica da Universidade Federal do Rio Grande do Norte (JGL, SOC, LHCN), e Centro de Endocrinologia de Natal (CEN) (JGL, MLCN, LHCN), Natal, $R N$; e Unidade de Endocrinologia e Diabetes do Hospital Agamenon Magalhães, SUS/Universidade de Pernambuco (FB, CHB, MP), Recife, PE.

Recebido em 17/06/05 Revisado em 28/11/05 Aceito em 15/12/05 
Uma vez diagnosticado, sua ressecção reverte os sintomas e pode curar a hipertensão arterial $(4,5)$. Porém, há considerável discussão a respeito de quais métodos laboratoriais seriam os mais apropriados para o diagnóstico do feocromocitoma (6-9).

Sabe-se que pacientes portadores de feocromocitomas grandes, maiores que $50 \mathrm{~g}$, podem apresentar pouca ou nenhuma alteração laboratorial (6). Com a finalidade de alertar sobre essa possível armadilha diagnóstica, os autores relatam caso de um paciente com grande feocromocitoma e valores normais de catecolaminas plasmáticas e metanefrinas urinárias.

\section{RELATO DO CASO}

Paciente do sexo masculino, 31 anos, procurou o serviço referindo que há 4 anos apresentara crise de dor lombar esquerda, com irradiação para hipogástrio, que cedeu com o uso de analgésicos. Dois anos após, voltou

Tabela 1. Exames laboratoriais pré-operatórios no paciente com grande feocromocitoma.

\begin{tabular}{lcc}
\hline Exame & Resultado & $\begin{array}{c}\text { Valores de } \\
\text { Referência }\end{array}$ \\
\hline $\begin{array}{l}\text { Glicose, uréia, creatinina, } \\
\text { ionograma, coagulograma, }\end{array}$ & Normais & - \\
hemograma & & - \\
Ácido vanili-mandélico $(\mathrm{VMA})$ & $37,0 \mathrm{mMol} / 24 \mathrm{~h} \leq 35 \mathrm{mMol} / 24 \mathrm{~h}$ \\
$\begin{array}{l}\text { Catecolaminas plasmáticas } \\
\text { - Epinefrina }(\mathrm{pg} / \mathrm{mL})\end{array}$ & 33,7 & $<140$ \\
- Norepinefrina $(\mathrm{pg} / \mathrm{mL})$ & 176,9 & $<1.400$ \\
- Dopamina $(\mathrm{pg} / \mathrm{mL})$ & 14,5 & $<30$ \\
$\begin{array}{l}\text { Metanefrinas urinárias } \\
\text { - Normetanefrina } \\
\quad \text { (mg/g de creatinina) }\end{array}$ & 0,21 & $<0,41$ \\
- Metanefrina & & \\
$\quad$ (mg/g de creatinina) & 0,01 & $<0,30$ \\
\hline
\end{tabular}

Tabela 2. Exames de imagem pré-operatórios no paciente com grande feocromocitoma.

\begin{tabular}{|c|c|}
\hline $\begin{array}{l}\text { Exame } \\
\text { Tomografia } \\
\text { computadorizada } \\
\text { de abdômen }\end{array}$ & $\begin{array}{l}\text { Resultado } \\
\text { Massa de } 9 \text { × } 8 \mathrm{~cm} \text {, com áreas de } \\
\text { provável necrose central envolvendo a } \\
\text { região de adrenal e pólo superior do rim } \\
\text { esq., invadindo e promovendo deformi- } \\
\text { dade na porção superior deste rim. }\end{array}$ \\
\hline $\begin{array}{l}\text { Ultrassonografia de } \\
\text { aparelho urinário } \\
\text { e adrenais }\end{array}$ & $\begin{array}{l}\text { Massa sólida, heterogênea, com área } \\
\text { cística central (necrose? hemorragia?), } \\
\text { medindo } 15 \times 9 \mathrm{~cm} \text {, localizada em flanco } \\
\text { esq., desviando a aorta medialmente e } \\
\text { a cauda pancreática anteriormente. }\end{array}$ \\
\hline $\begin{array}{l}\text { Cintilografia de } \\
\text { corpo inteiro } \\
\text { com MIBG-I }\end{array}$ & $\begin{array}{l}\text { Formação expansiva de contornos } \\
\text { irregulares e limites relativamente } \\
\text { precisos, situada em projeção do pólo } \\
\text { superior do rim esq., que apresenta área } \\
\text { de hipocaptação local em seu aspecto } \\
\text { súpero-lateral que deve corresponder } \\
\text { à área de necrose tumoral (figura 1). }\end{array}$ \\
\hline
\end{tabular}

a ter novo episódio de dor lombar, desta vez acompanhado de cefaléia, hipertensão arterial, rubor facial, sudorese e palidez cutânea. Há 1 ano, o quadro se repetiu, na presença de arritmia cardíaca e hipertensão arterial sustentada, tendo sido internado em outro hospital. Durante a internação, evoluiu com crise convulsiva, tratada com fenobarbital $100 \mathrm{mg} / \mathrm{dia}$. Ao exame físico, encontrava-se eupnéico, taquicárdico $(\mathrm{FC}=120$ bpm), ritmo cardíaco regular e $\mathrm{PA}=160 \times 100 \mathrm{mmHg}$. Ausculta pulmonar e exame do abdômen não apresentavam alterações. Foram colhidas amostras de sangue e de urina que imediatamente foram enviadas a laboratório para análise. O ácido vanilil-mandélico (VMA), as catecolaminas plasmáticas e as metanefrinas urinárias foram quantificados por Cromatografia Líquida de Alta Performance (HPLC). Os resultados dos exames laboratoriais e de imagem encontram-se nas tabelas 1 e 2 , respectivamente. Devido à possibilidade diagnóstica de feocromocitoma, foi realizada cintilografia com metaiodobenzilguanidina (MIBG-I131), que mostrou captação de grande massa em adrenal esquerda (figura l).

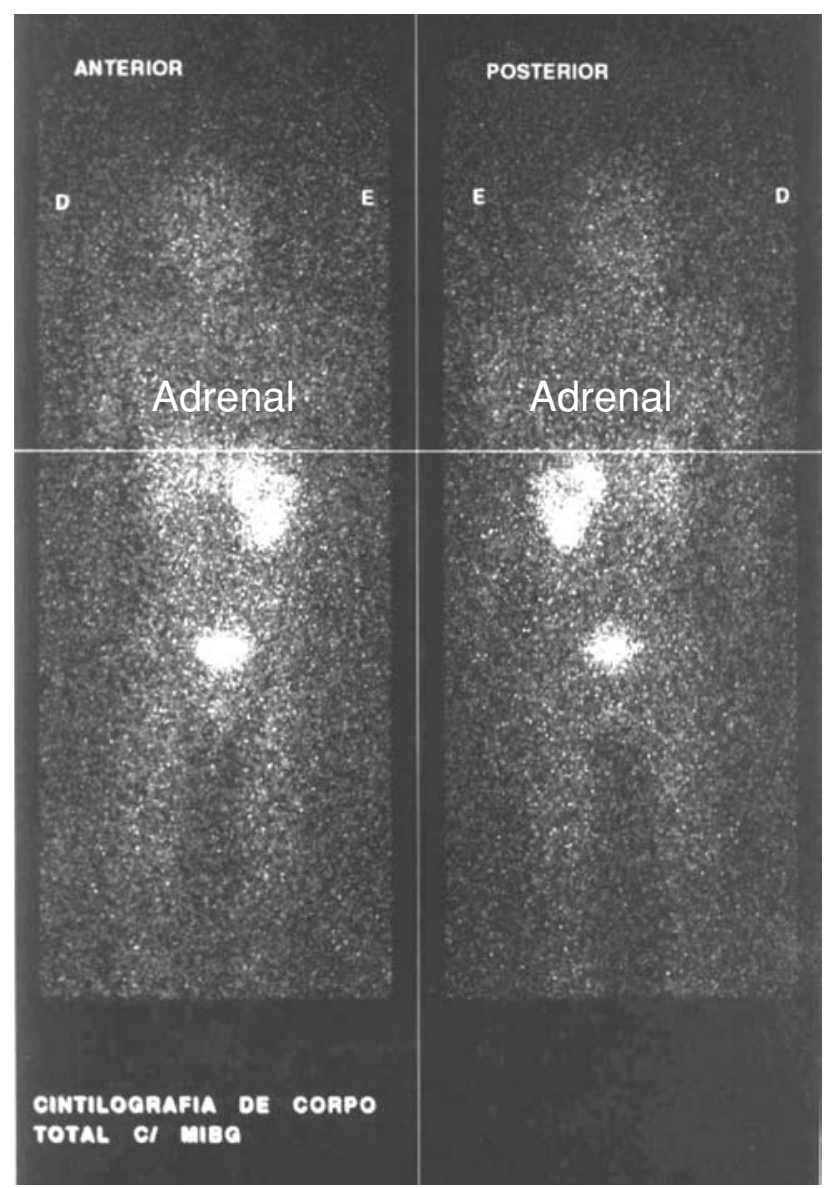

Figura 1. Cintilografia com meta-iodobenzilguanidina (MIBG). 
Foi feita a hipótese diagnóstica de feocromocitoma, tendo sido o paciente submetido a preparo pré-operatório com prazosin ( $5 \mathrm{mg} / \mathrm{dia}$ ), atenolol (100 mg/dia) e hidratação. Durante a cirurgia foi detectada invasão tumoral do rim esquerdo, optando por exérese do tumor adrenal e nefrectomia esquerda. Para controle da pressão arterial foram utilizados, durante a cirurgia, aproximadamente 8 litros de solução salina e 3 ampolas de nitroprussiato de sódio. A peça cirúrgica correspondia ao rim esquerdo aderido ao pólo superior a uma massa tumoral ovalada, aparentemente encapsulada, acastanhada e firmeelástica, medindo $11,0 \times 10,0 \times 9,0 \mathrm{~cm}$ e pesando $550 \mathrm{~g}$, com área central cística, de aspecto gelatinoso. O exame microscópico mostrou neoplasia adrenal compatível com feocromocitoma e parênquima renal com glomérulos apresentando celularidade conservada e congestão medular. $\mathrm{O}$ paciente não apresentou pico hipertensivo trans-operatório e evoluiu com normalização da pressão arterial após a cirurgia.

\section{DISCUSSÃO}

Relatamos caso atípico de feocromocitoma, apresentando sintomas típicos da doença e exame laboratorial isolado (VMA) minimamente alterado. Isso se configura numa armadilha diagnóstica que deve ser considerada sempre quando houver dicotomia entre dados clínicos e laboratoriais no diagnóstico do feocromocitoma.

Considerando a forma esporádica da doença (não-familial), as dosagens de metanefrinas totais urinárias têm, respectivamente, sensibilidade e especificidade na detecção do tumor de $88 \%$ e $89 \%$ (7). Para análise das catecolaminas plasmáticas, ainda na população com a forma esporádica, esses valores giram em torno de $92 \%$ e $72 \%$, respectivamente (7). O presente paciente apresentava hipertensão arterial mantida e o exame anátomo-patológico da massa retirada compatível com feocromocitoma, apesar de as catecolaminas séricas e as metanefrinas urinárias estarem normais.

Hipertensão arterial é a mais consistente manifestação do feocromocitoma (1). A tríade de sintomas formada por cefaléia, sudorese e taquicardia em paciente hipertenso tem sensibilidade de $90,9 \%$ e especificidade de $93,8 \%$ para essa doença (10). Apenas $8 \%$ dos casos são completamente assintomáticos e esses pacientes são usualmente os portadores de formas familiares da doença (diagnóstico precoce), ou com tumores grandes e císticos (6). O presente paciente não possuía história familiar positiva para feocromoci- toma e, provavelmente por ser oligossintomático, poderia tratar-se de caso com grande tumor cístico.

Parece não haver correlação entre os níveis de catecolaminas circulantes e a presença de hipertensão nos casos com feocromocitoma (11). Estes achados são confirmados também pelo presente caso, que apresentava hipertensão, apesar dos baixos níveis circulantes de catecolaminas.

Os exames de imagem mostraram padrão compatível com necrose hemorrágica do tumor. Essa necrose pode apresentar-se como um quadro de abdômen agudo, seguida de hipotensão e até choque e morte súbita (12). Isso decorre da marcada dilatação venosa e arterial decorrente da rápida diminuição das catecolaminas plasmáticas (13). Nosso paciente apresentou dois episódios de dor abdominal aguda, sendo que, no terceiro atendimento, foi observado quadro de hipertensão ao invés de hipotensão.

A ultrassonografia tem sensibilidade de $83-89 \%$ para a pesquisa de feocromocitoma $(14,15)$, enquanto que a especificidade para tumores adrenais em geral é de $60 \%$ (16). A tomografia computadorizada e a ressonância nuclear magnética têm sensibilidades $(98 \%$ e $100 \%$ respectivamente) e especificidades (92\% e $100 \%$ respectivamente) semelhantes (17). Pode-se atribuir a diferença entre as dimensões encontradas para o tumor entre os métodos de imagem (tabela 2) a algumas características operacionais. O caráter examinador dependente da ultrassonografia poderia sugerir a falta de fidedignidade na mensuração definitiva do tumor por esse método. Além disso, a tomografia computadorizada avaliou o paciente em cortes, podendo não estar considerando o maior diâmetro da massa tumoral. A cintilografia com MIBG-I ${ }^{131}$, apesar de apresentar elevada especificidade (95-100\%), tem sensibilidade de apenas $78 \%(18,19)$. A cintilografia positiva em nosso caso era compatível com o diagnóstico de feocromocitoma e justificou a indicação cirúrgica. A cintilografia com MIBG-I131, além de ser informativa quanto à localização anatômica, orienta sobre a caracterização funcional do tumor $(20,21)$. Como se trata de um tumor $\geq$ $8 \mathrm{~cm}$, com invasão de rim esquerdo, a cintilografia também é importante para excluir a o risco de metástase, doença extra-adrenal e multifocal (22).

No feocromocitoma, tumores maiores tendem a hemorragia, necrose e formação de cistos (23). Tumores maiores que $50 \mathrm{~g}$ metabolizam mais rapidamente as catecolaminas, levando ao paciente ser oligossintomático, apresentar níveis mais baixos de catecolaminas plasmáticas e metabólitos urinários mais elevados (6). Isso constitui, portanto, uma armadilha diagnóstica. Já tumores menores que $50 \mathrm{~g}$ tendem a produzir mais 
sintomas e serem mais facilmente diagnosticados devido aos valores elevados das catecolaminas plasmáticas (6). O presente caso se enquadra na descrição de portador de grande tumor (maior que $50 \mathrm{~g}$ ), com presença de necrose e hemorragia.

Feocromocitomas podem ser malignos se for detectada a presença de metástases (linfonodos, osso, pulmão, fígado) à cirurgia (22-25). $\mathrm{O}$ caso aqui descrito não apresentava metástases, tratando-se provavelmente de tumor benigno. Entretanto, evidências de malignidade podem ocorrer meses ou anos após a excisão cirúrgica de feocromocitoma aparentemente benigno (10). Devido ao tamanho tumoral e à idade do paciente, é importante segui-lo indefinidamente, por conta da alta probabilidade de recorrência tumoral (26).

A dosagem de metanefrinas livres no plasma apresenta altas sensibilidade e especificidade na detecção do feocromocitoma (7), mas esse exame não está disponível na grande maioria dos serviços. Mais recentemente têm-se enfatizado que o melhor teste diagnóstico varia com o risco prévio do paciente (27). Pacientes com alto risco de apresentarem feocromocitoma (portadores de neoplasias endócrinas múltiplas tipos $2 \mathrm{~A}$ ou $2 \mathrm{~B}$, síndrome de von HippelLindau, neurofibromatose tipo I, ou história familiar de paraganglioma), história passada de feocromocitoma, história familiar desse tumor, ou massa adrenal vascular são melhor diagnosticados por meio de teste com alta sensibilidade, mesmo com perda de especificidade (27). A dosagem das metanefrinas fracionadas plasmáticas livres, seguida de pesquisa do tumor por exame de imagem, parece ser o melhor protocolo para esse tipo de paciente (27).

Pacientes considerados de menor risco, como o aqui relatado, apresentando-se com hipertensão arterial resistente ao tratamento clínico, palpitações ou incidentalomas adrenais seriam melhor diagnosticados por meio de um exame com alta especificidade e aceitável sensibilidade, como a dosagem de metanefrinas e catecolaminas urinárias de 24 horas (27).

Devido à baixa especificidade das catecolaminas urinárias e também das plasmáticas $(75 \%$ e $72 \%$, respectivamente) (7), convém associar uma dessas dosagens à das metanefrinas totais em urina de 24 horas (especificidade 89\%) (7), da forma como foi realizado para o presente paciente.

As catecolaminas urinárias apresentam sensibilidade de 97\% (vs. 91\% para as plasmáticas) no que se refere ao diagnóstico de feocromocitoma esporádico (7). Metanefrinas plasmáticas livres, por sua vez, mostraram sensibilidade de $99 \%$ para a forma esporádica (7). Esses dados sugerem que fossem dosadas, em nosso paciente, as catecolaminas urinárias e metanefrinas plasmáticas livres. Porém, essas últimas são de difícil realização na grande maioria dos serviços no Brasil e, além disso, artigos enfatizando a importância das mesmas só foram publicados posteriormente ao caso $(7,27)$. Não há, portanto, condições de afirmar se, em nosso paciente, estes exames apresentariam alterações.

Concluindo, o presente relato enfatiza que, em pacientes com sintomas sugestivos de feocromocitoma, mesmo havendo valores normais de catecolaminas plasmáticas e metanefrinas urinárias, deve-se investigar a existência de tumor grande com metabolização intra-tumoral das catecolaminas ou tumor cístico quiescente.

\section{REFERÊNCIAS}

1. Young JWF. Pheochromocytoma: 1926-1993. Trends Endocrinol Metab 1993:4:122-7.

2. Omura M, Saito J, Yamaguchi K, Kakuta Y, Nishikawa T. Prospective study on the prevalence of secondary hypertension among hypertensive patients visiting a general outpatient clinic in Japan. Hypertens Res 2004:27:193-202.

3. Bech K, Hilden T. The frequency of secondary hypertension. Acta Med Scand 1975; 197:65-9.

4. Saegesser F. Cesar Roux (1857-1934) and his times. Rev Med Suisse Romande 1984; 104:403-64.

5. Mayo $\mathrm{CH}$. Paroxysmal hypertension with tumor of retroperitoneal nerve: Report of a case. JAMA 1927:89:1047-50

6. Crout JR, Sjoerdsma A. Turnover and metabolism of catecholamines in patients with pheochromocytoma. J Clin Invest 1964;43:94-102.

7. Lenders JW, Pacak K, Walther MM, Linehan WM, Mannelli $M$, Friberg $P$, et al. Biochemical diagnosis of pheochromocytoma: Which test is best? JAMA 2002;287:1427-34

8. Eisenhofer $G$, Lenders JW, Pacak K. Biochemical diagnosis of pheochromocytoma. Front Horm Res 2004; 31:76-106.

9. Kaltsas $G A$, Besser $G M$, Grossman $A B$. The diagnosis and medical management of advanced neuroendocrine tumors. Endocr Rev 2004;25:458-511.

10. Plouin PF, Chatellier G, Fofol I, Corvol P. Tumor recurrence and hypertension persistence after successful pheochromocytoma operation. Hypertension 1997:29:1133-9.

11. Bravo EL. Pheochromocytoma: An approach to antihypertensive management. Ann NY Acad Sci 2002;970: 1-10.

12. Gielchinsky I, Petty C, Dierdorff S. Treatment of hemorrhagic necrosis within a pheochromocytoma with symptoms of acute abdomen. Am Surg 1972;38:380-4. 
13. Mohamed HA, Aldakar MO, Habib N. Cardiogenic shock due to acute hemorrhagic necrosis of a pheochromocytoma: A case report and review of the literature. Can J Cardiol 2003; 19:573-6.

14. Witteles RM, Kaplan EL, Roizen MF. Sensitivity of diagnostic and localization tests for pheochromocytoma in clinical practice. Arch Intern Med 2000; 160:2521-4.

15. Lucon AM, Pereira MA, Mendonça BB, Halpern A, Wajchenbeg BL, Arap S. Pheochromocytoma: Study of 50 cases. J Urol 1997;157:1208-12.

16. Abrams HL, Siegelman SS, Adams DF, Sanders R, Finberg $\mathrm{HJ}$, Hessel SJ, et al. Computed tomography versus ultrasound of the adrenal gland: A prospective study. Radiology 1982;143:121-8.

17. Ilias I, Pacak K. Current approaches and recommended algorithm for the diagnostic localization of pheochromocytoma. J Clin Endocrinol Metab 2004:89:479-91.

18. Pacak K, Linehan WM, Eisenhofer $G$, Walther MM Goldstein DS. Recent advances in genetics, diagnosis, localization, and treatment of pheochromocytoma. Ann Intern Med 2001;134:315-29.

19. Bravo EL, Tagle R. Pheochromocytoma: State-of-the-art and future prospects. Endocr Rev 2003;24:539-53.

20. van Gils AP, Falke TH, van Erkel AR, Arndt JW, Sandler MP, van der Mey AG, et al. MR imaging and MIBG scintigraphy of pheochromocytomas and extra-adrenal functioning paragangliomas. Radiographics 1991;11:37-57.

21. Sisson JC, Frager MS, Valk TW, Gross MD, Swanson DP, Wieland DM, et al. Scintigraphic localization of pheochromocytoma. N Engl J Med 1981;305:12-7.
22. Pereira MAA, Souza BF, Freire DS. Pheochromocytoma. Arq Bras Endocrinol Metab 2004;48:751-75.

23. Robbins. Patologia estrutural e funcional. $6^{a}$ ed. Rio de Janeiro: Guanabara Koogan, 2000.

24. Shapiro B, Sisson JC, Lloyd R, Nakajo M, Satterlee W, Beierwaltes WH. Malignant phaeochromocytoma: Clinical, biochemical and scintigraphic characterization. Clin Endocrinol (Oxf) 1984;20:189-203.

25. Pattarino F, Bouloux PM. The diagnosis of malignancy in phaeochromocytoma. Clin Endocrinol (Oxf) 1996; 44:239-41.

26. Amar L, Servais A, Gimenez-Roqueplo AP, Zinzindohoue F, Chatellier G. Plouin PF. Year of diagnosis, features at presentation, and risk of recurrence in patients with pheochromocytoma or secreting paraganglioma. J Clin Endocrinol Metab 2005;90:2110-6.

27. Kudva YC, Sawka AM, Young WF, Jr. Clinical review 164: The laboratory diagnosis of adrenal pheochromocytoma: The Mayo Clinic experience. J Clin Endocrinol Metab 2003;88:4533-9.

\section{Endereço para correspondência:}

Josivan Gomes de Lima

Rua Joaquim Fabrício 233, ap, 1200

59012-340 Natal, RN

Fax: (84) 3211-6324

E-mail: josivan@usa.net 